Conclusions: Higher frequency of IL-17/IFN-g doublepositive Th17 cell with Pgp expression may be associated immunological and pharmacological factor for steroid resistance in LN.

Disclosure of Interest: None declared

DOI: 10.1136/annrheumdis-2018-eular.6457

\section{FRI0265 ANGIOGENIC T CELLS IN PRIMARY SJÖGREN'S SYNDROME: A DOUBLE-EDGED SWORD}

A. Alunno ${ }^{1}$, M. Manetti ${ }^{2}$, O. Bistoni ${ }^{1}$, S. Cipriani ${ }^{1}$, L. Ibba Manneschi ${ }^{2}$, R. Gerli ${ }^{1}$ ${ }^{1}$ Department of Medicine, Rheumatology Unit, University of Perugia, Perugia; ${ }^{2}$ Department of Experimental and Clinical Medicine, Section of Anatomy and Histology, University of Florence, Florence, Italy

Background: Angiogenic T cells (Tang) have been recently identified within colonies of endothelial progenitor cells (EPCs) as mediators of endothelial repair. Both Tang and EPCs are reduced in rheumatoid arthritis and this contributes to persistent endothelial damage and eventually increased cardiovascular risk. In primary Sjögren's syndrome (pSS), EPCs are expanded but no data are currently available about Tang.

Objectives: Aim of this study was to assess Tang (CD3 +CD31+CXCR4+) in peripheral blood (PB) and target organs of pSS as well as the association with EPCs (CD34+CD133+VEGFR-2+) and clinical and serological features of the disease.

Methods: Thirty-six pSS patients and 20 sex- and age-matched healthy donors (HD) were enrolled. Phenotipic analysis of peripheral blood mononuclear cells was performed by flow cytometry using FITC, Pe, Pe-Cy7 or AlexaFluor647 labelled anti-human CD3, CD31, CXCR4, CD4, CD8, CD28, CD34, CD133, VEGFR-2, and IL-17 antibodies. Minor salivary gland (MSG) biopsies from 8 pSS patients were studied and compared to samples from 12 patients with sicca symptoms and either non-specific chronic sialadenitis (NSCS) or normal parenchyma ( $n=6$ each). MSG sections were subjected to immunofluorescence staining to assess the presence of CD3 +CD31+CXCR4+Tang cells and the expression of the CXCR4-ligand CXCL12/SDF-1 chemokine.

Results: Circulating Tang were expanded in pSS compared to HD and were directly correlated to EPCs. Both Tang and EPCs directly correlated with disease activity as calculated with the EULAR Sjögren's syndrome disease activity index (ESSDAI). Over $60 \%$ of Tang lacked CD28 revealing a senescent phenotype. Only a small proportion of Tang displayed either CD4 or CD8, the majority of Tang being therefore CD4-CD8- double negative (DN). A subset of Tang produced IL17 and the highest proportion of IL-17-producing cells was observed among DN cells. Immunofluorescence analyses revealed the exclusive presence of infiltrating Tang cells along with increased expression of CXCL12/SDF-1 in pSS MSGs compared to either NSCS or normal MSGs.

Conclusions: Circulating Tang cells are expanded in pSS, display a senescent phenotype, are mainly CD4-CD8- DN and produce IL-17. Moreover, Tang cells home to and infiltrate MSGs in pSS, presumably through the SDF-1/CXCR4 chemotactic axis. Our data suggest that besides their positive effect together with EPCs in endothelial repair, Tang cells may contribute to disease pathogenesis.

Disclosure of Interest: None declared

DOI: 10.1136/annrheumdis-2018-eular.3062

\section{FRI0266 \\ CD16+ MONOCYTE SUBSETS IN PATIENTS WITH SYSTEMIC LUPUS ERYTHEMATOSUS, PRIMARY ANTIPHOSPHOLIPID SYNDROME, AND ANTIPHOSPHOLIPID SYNDROME WITH LUPUS ARE ASSOCIATED WITH SPECIFIC CLINICAL/SEROLOGICAL FEATURES OF THESE DISEASES}

C. Perez-Sanchez ${ }^{1}$, N. Barbarroja ${ }^{1}$, M.A. Aguirre ${ }^{1}$, P. Ruiz-Limon ${ }^{2}$, M.C. Abalos ${ }^{1}$, Y. Jimenez-Gomez ${ }^{1}$, I. Arias de la Rosa ${ }^{1}$, P. Segui ${ }^{3}$, R. Ortega ${ }^{1}$, E. Collantes ${ }^{1}$, A. Escudero ${ }^{1}$, L. Le Lann ${ }^{4}$, C. Jamin ${ }^{4}$, M. Alarcon ${ }^{5}$, J.O. Pers ${ }^{4}$, C. Lopez-Pedrera ${ }^{1}$, on behalf of PRECISESADS Clinical Consortium and Precisesads Flow Cytometry Collaborative Group. ${ }^{1}$ GC-5/Rheumatology, Reina Sofia Hospital-imibic, Cordoba; ${ }^{2}$ FIMABIS-Virgen de la Victoria Hospital, Malaga; ${ }^{3}$ Radiology, Reina Sofia Hospitalimibic, Cordoba, Spain; ${ }^{4}$ Université de Brest, Brest, France; ${ }^{5}$ Genyo, Granada, Spain

Objectives: This study, developed within the IMI-JU project PRECISESADS framework, aimed to determine the enrichment on CD14 +and CD16+monocyte subpopulations in SLE, APS and APS +SLE patients, and to investigate their role in the pathogenesis of these diseases.

Methods: The frequencies of monocyte subpopulations in the peripheral blood of 54 healthy donors and 46 SLE patients included in the PRECISESADS study (preliminary data) were determined by flow cytometry. A second cohort of 21 APS +SLE patients and a third cohort of 19 APS patients were included. Clinical profile, proinflammatory circulating mediators, and peroxide levels were analysed. Carotid intima media thickness (CIMT) was evaluated as atherosclerosis marker

Results: The CD14 +CD16+ (non-classical) monocyte subset was reduced in SLE patients. Their frequencies were negatively associated with the positivity for anti-dsDNA, anti-SSB, anti-SSA and anti-U1RNP autoantibodies, as well as with renal involvement, which might reflect a recruitment process of this subset in renal tissues. Correlation studies further indicated a link between the reduced frequency of non-classical monocytes and increased levels of circulating inflammatory mediators. Conversely, SLE patients with a previous history of thrombosis displayed a significant increase in circulating CD14 +CD16+monocytes in relation to those without previous thrombotic manifestations. These results prompted us to evaluate the proportion and profile of this inflammatory subtype in parallel cohorts of SLE +APS and APS patients, on which thrombosis constitute the main clinical disorder

SLE +APS patients showed enrichment in intermediate (CD14 ++CD16+) and non-classical monocytes, associated with the positivity for anti-dsDNA antibodies and the presence of atheroma plaques. Correlations among the frequency of those monocyte subsets and inflammatory mediators, and circulating cytokines, were also demonstrated. Yet, SLE +APS patients with renal involvement dis played reduced proportion of circulating intermediate and non-classical monocytes, suggesting that, as in the case of SLE patients, circulating CD16 +cells might have migrated to the kidney.

In APS patients we also saw enrichment of the CD16 +inflammatory subsets associated to recurrent thrombotic events and a pathologic CIMT, pointing out that such subsets are associated with CVD. The scores of various markers related to autoimmunity, oxidative stress and prothrombotic molecules further correlated with the proportions of intermediate and non-classical monocytes.

Conclusions: Circulating CD16 +monocytes might constitute an important subpopulation of proinflammatory cells, whose frequency might identify APS, APS +SLE and SLE patients suffering thrombosis, atherosclerosis and organ involvement.

Acknowledgements: Supported by: EU/EFPIA-IMI-PRECISESADS (n 115565), Instituto de Salud Carlos III (FIS PI15/1333) and Junta Andalucia (CTS7940).

Disclosure of Interest: None declared

DOI: 10.1136/annrheumdis-2018-eular.3265

FRI0267

CHARACTERISATIONOF THE MOLECULAR PROFILE OF ALTERED GENES AND PATHWAYS IN MONOCYTES OF ANTIPHOSPHOLIPID SYNDROME PATIENTS RELATED TO THEIR ATHEROTHROMBOTIC STATUS. EFFECTS OF IN VIVO UBIQUINOL SUPPLEMENTATION

C. Perez-Sanchez ${ }^{1}$, M.A. Aguirre ${ }^{1}$, L. Perez-Sanchez ${ }^{1}$, M. Luque-Tevar ${ }^{1}$ N. Barbarroja ${ }^{1}$, P. Ruiz-Limon ${ }^{1}$, Y. Jimenez-Gomez ${ }^{1}$, I. Arias de la Rosa ${ }^{1}$, M. C. Abalos ${ }^{1}$, P. Segui ${ }^{1}$, J.M. Villalba ${ }^{1}$, E. Collantes ${ }^{1}$, M.J. Cuadrado ${ }^{2}$, C. LopezPedrera'. ${ }^{1}$ IMIBIC/Reina Sofia Hospital/University of Cordoba, Córdoba, Spain; ${ }^{2} \mathrm{St}$ Thomas Hospital, London, UK

Objectives: This study was undertaken to: 1. Characterise monocytes molecular profile of altered genes and pathways involved in the pathology of APS. 2. To evaluate the role of antiphospholipid antibodies in the regulation of these processes. 3. To investigate the short-term effects of in vivo ubiquinol (reduced coenzyme Q10 [Qred]) supplementation on the modulation of genes related to inflammation and thrombosis in this autoimmune disorder.

Methods: Monocytes from peripheral blood of 60 subjects, including 30 APS patients and 30 healthy donors (HDs) were purified by negative immunomagnetic selection (Miltenyi). Total RNA was extracted from 6 subjects -as exploratory cohort- and microarray studies were performed in an Agilent G4112F platform (Whole Human Genome Microarray 44 k). Functional categorization of the altered gene signature and molecular pathways and networks was carried out using the Ingenuity Pathway Analysis Software (IPA). The most differentially expressed genes were validated by RT-PCR in monocytes purified from all the subjects recruited to the study. Correlation and association studies were performed with clinical and analytical variables. The effects of antiphospholipid antibodies (aPL) were also evaluated by in vitro studies. The short-term effects of in vivo Qred supplementation on the monocyte gene profile were further analysed.

Results: Gene expression array identified 518 altered genes in monocytes from APS patients in relation to the control group ( $p<0,05$ and fold change $>2$ ). IPA analysis showed that the main canonical pathways integrated by these genes were leukocyte adhesion, diapedesis and extravasation signalling, interleukin and cytokine signalling, as well as oxidative stress production and antioxidant response. This analysis further identified that the most relevant diseases in which these altered genes are involved were inflammatory and cardiovascular diseases $(44 \%)$, as well as reproductive $(42 \%)$, neurological $(11 \%)$, renal $(1 \%)$ and ophthalmic diseases $(2 \%)$. The alteration of several of these genes was validated by RTPCR and protein analysis, and associated to clinical parameters of APS patients including thrombotic recurrences and early atherosclerosis. In vitro studies 
demonstrated the specific modulation of several genes by direct effect of aPLs. In vivo Qred supplementation of APS patients for one month significantly improved the monocytes' atherothrombotic gene profile, reversing the altered expression of a number of genes related to thrombosis, atherosclerosis, inflammation, oxidative stress, and intracellular signalling

Conclusions: 1. Gene expression profile allowed the identification of relevant genes and pathways altered in monocytes of APS patients, which are associated with the pathogenesis of the disease and modulated, at least partially, by aPLs. 2. We have branded novel and specific mRNAs related to CVD in APS monocytes, further modified by effect of in vivo Qred supplementation.

Acknowledgements: Funded by JA (CTS-7940) and Ministry of Health (ISCIII, PI15/01333 and RIER RD16/0012/0015) cofinanced with FEDER funds.

Disclosure of Interest: None declared

DOI: 10.1136/annrheumdis-2018-eular.6371

\section{FRI0268 GLUCOCORTICOID-INDUCED LEUCINE ZIPPER (GILZ) REPRESENTS A CHECKPOINT LIMITING TYPE I INTERFERON (IFN) PRODUCTION IN SLE}

C.T. Nataraja, E.F. Morand, J. Thomas, J. Harris, S.A. Jones. Department of Medicine, Monash University, Melbourne, Australia

Background: Glucocorticoids (GC) remain the mainstay of treatment in Systemic Lupus Erythematosus (SLE). Type I interferons (IFN), produced by plasmacytoid dendritic cells ( $p D C$ ) in response to Toll-Like receptors (TLR) ligands, are critical to SLE pathogenesis, but are not suppressed by GC. Glucocorticoid-Induced Leucine Zipper (GILZ) is an endogenous anti-inflammatory protein induced by GC. Beaulieu et al., 2010 However, whether GILZ regulates IFN production in SLE is not known.

Objectives: To test the hypothesis that GILZ inhibits the production of Type I IFN in SLE.

Methods: We performed in vitro analysis on $\mathrm{pDC}$ and bone marrow-derived $\mathrm{DC}$ (BMDC), and in vivo studies, of WT and GILZ-/- mice using stimuli of TLR7 (Imiquimod), TLR7/8 (Resiquimod) and TLR9 (CpG). IFN was measured using a IFN luciferase assay, and other cytokines with ELISA. IFN-stimulated gene signatures (ISG) were measured using qPCR. To determine whether GILZ regulates IFN in human SLE, we mined a public gene expression dataset GSE10325. Becker et al., 2013

Results: Deletion of GILZ resulted in excess pDC secretion of IFN in response to TLR7 $(p=0.0012)$ and TLR9 $(p=0.01)$ stimulation, and BMDC secretion of IFN, IL6 and TNF $\alpha$ in response to TLR7 $(p=0.0039,0.017,0.033), T L R 7 / 8(p=0.001$ $<0.0001,<0.0001)$ and TLR9 $(p=0.005,<0.0001,0.0034)$ stimulation respectively. Dexamethasone (DEX) induced GILZ in WT pDC and BMDC, and TLR stimulation suppressed GILZ expression in BMDC, but TLR-stimulated GILZ-/- cell failed to suppress IFN in response to DEX. Moreover, GILZ deficiency was associated with increased ISG in naïve spleen cells, naïve BMDCs and TLR7/9 stimulated pDC of GILZ-/- mice compared to WT mice. Correspondingly, increased IFN was seen in GILZ-/- mice in response to TLR7/8 stimulation in vivo. In GSE10325, we show that lower expression of GILZ was associated with high ISG (IFI44, IFI44L, RSAD2, IFI27) ( $\mathrm{p}=0.0021)$ in SLE patient peripheral blood B cells, and GILZ mRNA was negatively correlated with IFN signature $(r=-0.63, p=0.017)$ which in turn positively correlated with disease activity (SLEDAI2k) $(r=0.77, p=0.002)$.

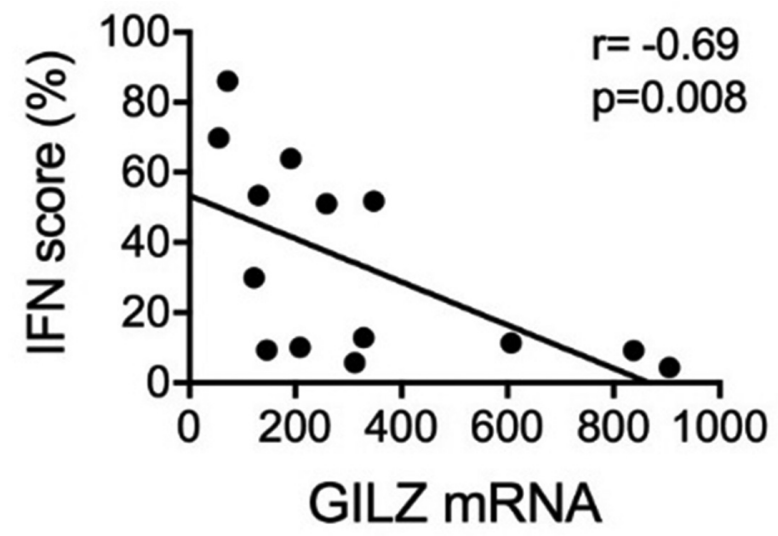

Conclusions: GILZ is an endogenous regulator of increased IFN production in response to TLR stimulation in vitro and in mice, and is negatively correlated with ISG in human SLE. This suggests that GILZ negatively regulates type I IFN production and GILZ based therapy may be a potential therapeutic strategy that could reduce steroid dependence in SLE.
REFERENCES:

[1] Beaulieu E, Ngo D, Santos L, Yang YH, Smith M, Jorgensen C, ... Morand EF. Glucocorticoid-induced leucine zipper is an endogenous antiinflammatory mediator in arthritis. Arthritis Rheum 2010;62(9):2651-2661. doi:10.1002/art.27566

[2] Becker AM, Dao KH, Han BK, Kornu R, Lakhanpal S, Mobley AB, Davis LS. SLE peripheral blood B cell, T cell and myeloid cell transcriptomes display unique profiles and each subset contributes to the interferon signature. PLoS One 2013;8(6):e67003. doi:10.1371/journal.pone.0067003

Acknowledgements: NHMRC

Disclosure of Interest: None declared

DOI: 10.1136/annrheumdis-2018-eular.1847

\section{FRI0269 DEFICIENCY OF GLUCOCORTICOID-INDUCED LEUCINE ZIPPER (GILZ) DISINHIBITS IFN PATHWAYS AND EXACERBATES NEPHRITIS IN THE LYN-DEFICIENT MURINE MODEL OF LUPUS}

C.T. Nataraja, E.F. Morand, J. Thomas, J. Harris, S.A. Jones. Department of Medicine, Monash University, Melbourne, Australia

Background: Systemic lupus erythematosus (SLE) is a multi-system autoimmune disease of unknown etiology. Lyn-deficient mice develop lupus-like autoimmunity due to hyperactive $B$ cells resulting in excess IL-6 production, and cyclical exacerbation of inflammation by further activation of B and T cells. Tsantikos et al., 2010 C57BL/6 mice deficient in glucocorticoid-induced leucine zipper (GILZ), an intracellular protein involved in glucocorticoid effects on immunity, develop lupus-like autoimmunity and excess B cell activation. Jones et al., 2016. However, the effect of GILZ in lupus-prone mice is unknown.

Objectives: We aim to test the hypothesis that GILZ modulates autoimmunity in the lyn deficient murine model of lupus.

Methods: We generated GILZ-deficient mice on a lyn-deficient background (GILZ-lyn double knock out (DKO)) and compared them to WT and lynKO mice. The effects of GILZ deficiency on spleen weight, nephritis, Type I interferoninduced genes (ISG) and autoantibodies were examined.

Results: We observed heightened lupus-like autoimmunity in GILZ-lyn DKO mice, compared to LynKO, that include increased spleen weights $(p=0.041)$ and more severe glomerulonephritis, especially segmental necrosis. A panel of ISG (ifi44, usp18, oas3, cxcl10, isg15, $m \times 1$, irf7, stat1 and ifit3) and an overall ISG score was significantly increased $(p=0.0023)$ in GILZ-lyn DKO mice compared to LynKO. In contrast, serum autoantibodies (dsDNA, Sm, histone, Jo-1, Scl-70, SSA, SSB, U1RNP, Ro52,) were not increased in GILZ-lyn DKO mice compared to LynKO mice.

\section{Glomerular segmental necrosis}

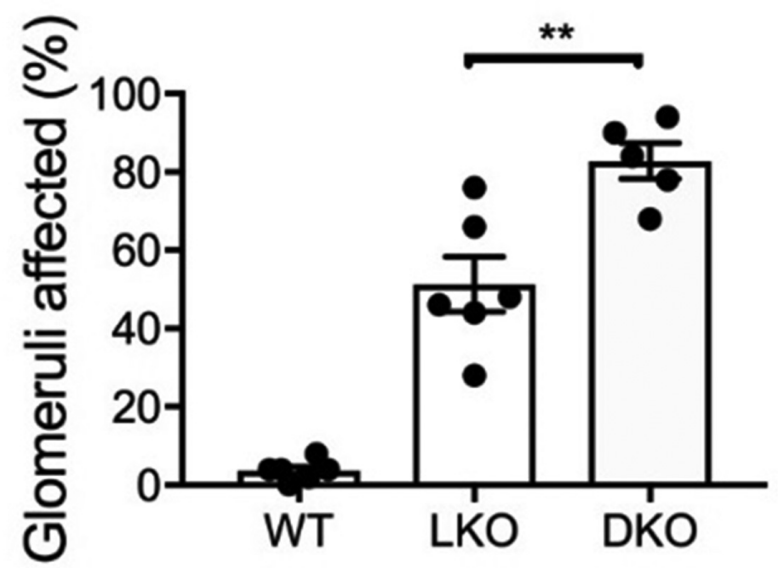

Conclusions: In LynKO lupus-prone mice, spleen weight, glomerulonephritis and ISG profiles were significantly exacerbated by GILZ deficiency, while autoantibody titres were unaffected. This suggests that endogenous GILZ exerts an inhibitory effect on IFN pathways, in this lupus model. Therefore, GILZ potentially regulates the cycle of inflammation in SLE by inhibiting IFN responses 